\title{
SIMULAÇÕES POR ELEMENTOS FINITOS APLICADAS AOS ENSAIOS DE UM TRANSFORMADOR EM PRÉ-AULA DE PRÁTICA LABORATORIAL
}

\author{
Thyago Chrystiann da Silva Lopes - thyago.chrystiann.1996@gmail.com \\ Victor de Paula Brandão Aguiar - victor@ufersa.edu.br \\ Adriano Aron Freitas de Moura - adrianoaron@ufersa.edu.br \\ Universidade Federal Rural do Semi-Árido (UFERSA), Departamento de Engenharia e \\ Tecnologia (DET) \\ Av. Francisco Mota, 572, Campus Leste, Prédio do CE II \\ 59625-900 - Mossoró - Rio Grande do Norte
}

Wilkley Bezerra Correia - wilkley@dee.ufc.br

Universidade Federal do Ceará (UFC), Laboratório de Sistemas Motrizes (LAMOTRIZ)

Campus do Pici, Departamento de Engenharia Elétrica, Bloco 705, Caixa Postal 6001

60440-554 - Fortaleza - Ceará

Resumo: Neste artigo é apresentada a aplicação do método dos elementos finitos para a determinação de parâmetros de um transformador, no qual o mesmo foi previamente analisado por meio de procedimentos experimentais, chamados de ensaios. No método apresentado é levada em consideração a conexão com circuitos elétricos, o funcionamento no domínio do tempo, bem como as características do material do núcleo do transformador nas simulações pelo método dos elementos finitos. As simulações oferecem uma boa precisão na obtenção dos dados que os ensaios fornecem e uma comparação entre resultados de simulação e resultados experimentais podem ser realizadas, mostrando a técnica da sala de aula invertida em aula práticas laboratoriais e a motivação da previsão dos resultados experimentais.

Palavras-chave: Sala de aula invertida. Ensaio a vazio. Ensaio de curto-circuito. Simulação em $3 D$.

\section{INTRODUÇÃO}

$\mathrm{Na}$ aprendizagem ativa, principalmente na abordagem sala de aula invertida, o momento de preparação a aula, ou a primeira exposição a determinado assunto, é conhecida como préaula e sua importância é destacada em diversos campos do conhecimento e universidades na aprendizagem de conhecimento teórico e prático (ELMÔR FILHO et al, 2019). Em disciplinas práticas em laboratórios, a aprendizagem ativa está presente quando o professor disponibiliza como pré-aula o roteiro da prática para leitura. Lembrando que, pelo diagrama de Venn, o envolvimento do estudante no ato de aprender requer além da aprendizagem ativa, também a motivação (ELMÔR FILHO et al, 2019). Portanto inovar na pré-aula é mandatório.

Assim, neste trabalho trataremos a ideia de que a aula que antecede a aula prática seria uma aula teórica de simulação dos fenômenos envolvidos e que serviria de um momento inicial e de introdução ao tema a ser abordado na aula prática dentro do laboratório. Ademais, os métodos para esta simulação e o efeito teórico de observação e comparação com resultados 
obtidos em práticas laboratoriais podem utilizar métodos de ponta na ciência moderna e neste conjunto de ações deve haver preocupação em aplicar o método científico para o futuro engenheiro. Este tipo de abordagem abre a possibilidade da aprendizagem ativa dentro de um modelo híbrido de aprendizagem, com estudos à distância como pré-aula e, na sequencia, uma abordagem prática dentro do laboratório da instituição (ELMÔR FILHO et al, 2019).

Diante da necessidade de análises precisas em máquinas elétricas e transformadores em conjunto com os avanços em tecnologias computacionais alcançados recentemente, o emprego de métodos numéricos tornou-se indispensável (BASTOS E SADOWSKI, 2013).

$\mathrm{O}$ método dos elementos finitos (MEF) é um método numérico que possibilita a análise de máquinas elétricas de geometrias complexas (SADIKU, 2000). Tal procedimento é possível em virtude de que no MEF é realizada a subdivisão da geometria em pequenas partes, chamadas de elementos, onde são aplicadas as formulações matemáticas para a resolução dos fenômenos físicos desejados. A precisão dos resultados obtidos por meio do MEF dá-se em função da possibilidade em considerar inúmeros aspectos presentes em um fenômeno físico. Para um transformador, o MEF possibilita levar em consideração a sua alimentação por meio de circuitos elétricos, a operação no domínio do tempo, bem como o comportamento não linear do núcleo.

Este artigo possui como objetivo programar um simulador de MEF de forma complementar aos ensaios empregados em transformadores, servindo como ferramenta comparativa entre resultados medidos e simulados em uma modelagem em três (3) dimensões (3D).

\section{ENSAIOS EM TRANSFORMADORES}

Os ensaios realizados em um transformador são procedimentos experimentais que visam determinar seus parâmetros, possibilitando a montagem de seu circuito equivalente, indicado na Figura 1. Em transformadores, os principais ensaios empregados para este objetivo são o ensaio a vazio (EAV) e o ensaio de curto-circuito (ECC).

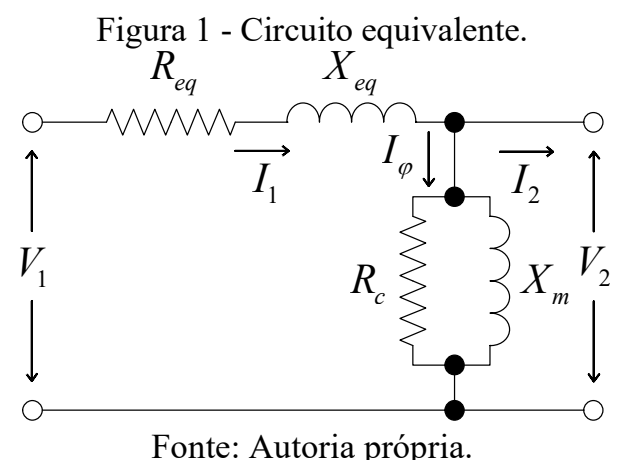

Na Figura $1, R_{e q}$ e $X_{e q}$ representam, respectivamente, a resistência e reatância equivalente dos enrolamentos, $R_{c}$ é a resistência do núcleo e $X_{m}$ é a reatância de magnetização. $V_{1}, V_{2}, I_{l}$ e $I_{2}$ são as tensões e correntes dos enrolamentos primário e secundário, enquanto $I_{\varphi}$ é a corrente de excitação. Os valores de tensão, $V_{E A V}$ e $V_{E C C}$; corrente, $I_{E A V}$ e $I_{E C C}$; e potência ativa, $P_{E A V}$ e $P_{E C C}$; no EAV e ECC, respectivamente, são obtidos por meio de medição nos terminais do enrolamento primário, sendo utilizados para determinar os componentes do circuito equivalente por meio das equações presentes no Quadro 1. 
Quadro 1 - Parâmetros do circuito equivalente.

\begin{tabular}{|c|c|}
\hline$R_{c}=\frac{V_{E A V}^{2}}{P_{E A V}}$ & $R_{e q}=\frac{P_{E C C}}{I_{E C C}^{2}}$ \\
\hline$X_{m}=\frac{1}{\sqrt{\left(\frac{I_{E A V}}{V_{E A V}}\right)^{2}-\left(\frac{1}{R_{c}}\right)^{2}}}$ & $X_{e q}=\sqrt{\left(\frac{V_{E C C}}{I_{E C C}}\right)^{2}-R_{e q}^{2}}$ \\
\hline
\end{tabular}

Fonte: Carvalho (2008).

\section{MÉTODO DOS ELEMENTOS FINITOS}

O MEF consiste em procedimentos computacionais cujo objetivo é resolver, por meio de métodos numéricos, problemas de valores de contorno (PVC) que descrevem fenômenos físicos. Um PVC consiste em equações diferenciais (ED) que atuam em uma região, $\Omega$, chamada domínio, submetidas a restrições que atuam na fronteira, $\Gamma$, desse domínio, denominadas condições de fronteira. A resolução do PVC é feita aplicando o método residual de Galerkin, no qual se determina uma solução aproximada.

\subsection{Definição do problema e discretização do domínio}

O PVC que descreve o funcionamento do transformador é caracterizado pela ED apresentada na Equação (1) (RENYUAN et al., 2000), submetida as condições de fronteira definidas nas Equações (2), (3), (4) e (5). Essa ED atua sobre o domínio $\Omega$ e descreve o comportamento do campo em função de uma fonte externa.

$$
\begin{aligned}
\nabla \times(v \nabla \times \vec{A})-\nabla(v \nabla \cdot \vec{A}) & +\sigma \frac{\partial \vec{A}}{\partial t}-\frac{N_{e s p}}{S_{d o m}} I_{c i r} \vec{e}=\overrightarrow{0} \quad \text { em } \Omega \\
\vec{n} \times \vec{A} & =\overrightarrow{0} \quad \text { em } \Gamma \\
(v \times \vec{A}) \times \vec{n} & =\overrightarrow{0} \quad \text { em } \Gamma \\
\nabla \cdot \vec{A} & =0 \quad \text { em } \Gamma \\
\vec{A} \cdot \vec{n} & =0 \quad \text { em } \Gamma
\end{aligned}
$$

onde $\sigma$ é a condutividade do domínio $\Omega, v$ é a relutividade magnética, $\vec{n}$ é o vetor normal à superfície da fronteira $\Gamma$ e $\partial / \partial t$ é a derivada parcial em relação ao tempo.

A Equação (1), bem como suas condições de fronteira, são obtidas a partir das equações de Maxwell na condição quasi-estatica com o potencial vetor magnético, $\vec{A}$, mediante a condição de Coulumb (BÍRÓ; PREIS; PAUL, 1999). A variável $I_{c i r}$ representa a corrente proveniente de um circuito elétrico conectado a um enrolamento do transformador, e possibilita o acoplamento da equação do campo com equações de circuito elétrico. O vetor densidade de enrolamento, $\vec{e}$, é sempre tangencial ao domínio do enrolamento, e define a direção da corrente. As variáveis $N_{e s p}$ e $S_{d o m}$ são o número de espiras e a seção reta do domínio do enrolamento, respectivamente. 


\section{COBENGE (C) COBENCE 2020

\section{"Os desafios para formar hoje o engenheiro do amanhã"}

01 a 03 de dezembro

\section{Evento On-line}

Figura 2 - a) Domínio e malha do PVC. b) Tetraedro linear.

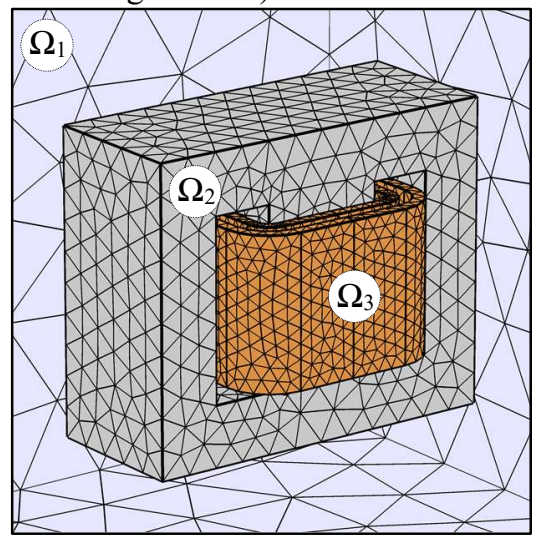

a)

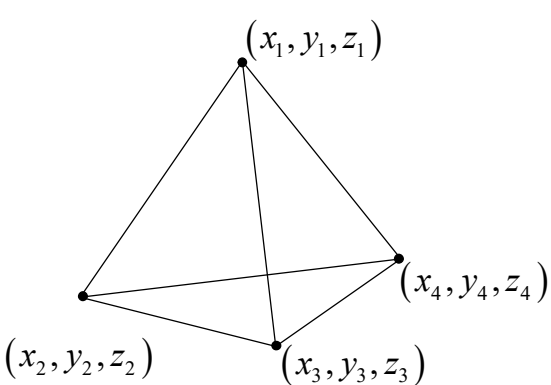

b)

Fonte: Autoria própria.

$\mathrm{Na}$ Figura 2a, está representado o domínio $\Omega$ do PVC em questão, que pode ser subdividido nos domínios $\Omega_{1}$, representando o ar que envolve o transformador; $\Omega_{2}$, que representa o núcleo do transformador; e $\Omega_{3}$, caracterizando os enrolamentos. O domínio do PVC é discretizado conforme a Figura 2a, utilizando a junção de tetraedros lineares, Figura $2 \mathrm{~b}$, interligados por meio de seus vértices, formando uma malha, na qual cada tetraedro é um elemento da malha e cada vértice é um nó da malha. O potencial vetor magnético é definido nos nós de um elemento $e$ de acordo com a Equação (6).

$$
\vec{A}^{e}=\vec{x} A_{x}^{e}+\vec{y} A_{y}^{e}+\vec{z} A_{z}^{e}=\vec{x} \sum_{i=1}^{4} N_{i} A_{x i}^{e}+\vec{y} \sum_{i=1}^{4} N_{i} A_{y i}^{e}+\vec{z} \sum_{i=1}^{4} N_{i} A_{z i}^{e}
$$

onde $A_{x}^{e}, A_{y}^{e}$ e $A_{z}^{e}$ são as componentes de $\vec{A}^{e}$, e $A_{x i}^{e}, A_{y i}^{e}$ e $A_{z i}^{e}$ são seus respectivos valores no nó $i$ de um elemento $e$. A função de forma, $N_{i}$, é definida como:

$$
N_{i}(x, y, z)=\frac{1}{6 V}\left(\alpha_{i}+\beta_{i} x+\gamma_{i} y+\delta_{i} z\right)
$$

onde os coeficientes $\alpha_{i}, \beta_{i}, \gamma_{i}$ e $\delta_{i}$ são obtidos a partir da relação na Equação (8), e o volume do elemento, $V$, é definido na relação da Equação (9).

$$
\begin{gathered}
{[M]^{-1}=\left[\begin{array}{cccc}
1 & x_{1} & y_{1} & z_{1} \\
1 & x_{2} & y_{2} & z_{2} \\
1 & x_{3} & y_{3} & z_{3} \\
1 & x_{4} & y_{4} & z_{4}
\end{array}\right]^{-1}=\frac{1}{6 V}\left[\begin{array}{cccc}
\alpha_{1} & \alpha_{2} & \alpha_{3} & \alpha_{4} \\
\beta_{1} & \beta_{2} & \beta_{3} & \beta_{4} \\
\gamma_{1} & \gamma_{2} & \gamma_{3} & \gamma_{4} \\
\delta_{1} & \delta_{2} & \delta_{3} & \delta_{4}
\end{array}\right]} \\
6 V=\operatorname{det}[M]
\end{gathered}
$$

\subsection{Método de Galerkin}

A aplicação do Método de Galerkin dá-se multiplicando a própria ED da Equação (1) por uma função teste, $W$. Realiza-se então a integração por todo domínio da ED, na qual a integral resultante possui valor nulo (BASTOS E SADOWSKI, 2003), conforme a Equação (10). 


\section{COBENGE 2020

\section{"Os desafios para formar hoje o engenheiro do amanhã"}

\section{Evento On-line}

$$
\int_{\Omega} W\left[\nabla \times(v \nabla \times \vec{A})-\nabla(v \nabla \cdot \vec{A})+\sigma \frac{\partial \vec{A}}{\partial t}-\frac{N_{e s p}}{S_{d o m}} I_{c i r} \vec{e}\right] d \Omega=0
$$

Para $W$ definido como a transposta da função de forma nos vértices $j, N_{j}^{T}$, tem-se:

$$
\begin{gathered}
v \int_{\Omega} \nabla \times N_{j}^{T} \cdot \nabla \times N_{i} d \Omega A^{e}-\int_{\Gamma}(v \nabla \times \vec{A} \times \vec{n}) \cdot N_{j}^{T} d \Gamma+v \int_{\Omega} \nabla \cdot N_{j}^{T} \nabla \cdot N_{i} d \Omega A^{e}-\ldots \\
\ldots-\int_{\Gamma} v \nabla \cdot N_{j}^{T} \vec{A} \cdot \vec{n} d \Gamma+\sigma \int_{\Omega} N_{j}^{T} \cdot N_{i} d \Omega \frac{\partial A^{e}}{\partial t}-\frac{N_{e s p}}{S_{\text {dom }}} \int_{\Omega} N_{j}^{T} \cdot \vec{e} d \Omega I_{c i r}=0
\end{gathered}
$$

Conforme as Equações (2) a (5), a forma matricial da Equação (11) é definida em:

$$
v[S]\{A\}+[T]\left\{\frac{\partial A}{\partial t}\right\}-[D]\left\{I_{\text {cir }}\right\}=0
$$

onde matrizes $[S],[T]$ e $[D]$ em um elemento $e$ são definidas como:

$$
\begin{gathered}
S_{i j}^{e}=\int_{\Omega} \nabla \times N_{j}^{T} \cdot \nabla \times N_{i} d \Omega+\int_{\Omega} \nabla \cdot N_{j}^{T} \nabla \cdot N_{i} d \Omega \\
T_{i j}^{e}=\sigma \int_{\Omega} N_{j}^{T} \cdot N_{i} d \Omega \\
D_{j}^{e}=\frac{N_{\text {esp }}}{S_{\text {dom }}} \int_{\Omega} N_{j}^{T} \cdot \vec{e} d \Omega
\end{gathered}
$$

\subsection{Acoplamento da equação de campo com equações de circuito elétrico}

O acoplamento da equação do campo com a equação do circuito elétrico, determinadas nas Equações (1) e (16), respectivamente, permite uma análise precisa do transformador ao considerar parâmetros práticos utilizados nos ensaios, tais como a tensão aplicada a um enrolamento, $V_{\text {cir }}$, e a corrente do circuito, $I_{\text {cir }}$. Na Equação (16), $R$ é a resistência de um enrolamento conectado ao circuito elétrico, definida na Equação (17);

$$
\begin{gathered}
V_{c i r}=R I_{c i r}+\frac{N_{e s p}}{S_{d o m}} \int_{\Omega} \frac{\partial \vec{A}}{\partial t} \cdot \vec{e} d \Omega \\
R=\frac{N_{e s p} l}{\sigma_{e n r} S_{e n r}}
\end{gathered}
$$

onde $l, \sigma_{e n r}$ e $S_{e n r}$ são o comprimento médio, a condutividade e a seção reta do enrolamento, respectivamente. Aplicando o método de Galerkin, a Equação (16) torna-se:

$$
\frac{N_{e s p}}{S_{\text {dom }}} \int_{\Omega} N_{j}^{T} \cdot N_{i} \cdot \vec{e} d \Omega \frac{\partial A^{e}}{\partial t}+R I_{c i r}-V_{c i r}=0
$$

A forma matricial da Equação (18) é: 


$$
[E]\left\{\frac{\partial A}{\partial t}\right\}+[R]\left\{I_{c i r}\right\}-\left\{V_{c i r}\right\}=0
$$

A matriz $[E]$ em um elemento $e$ é dada por:

$$
E_{i j}^{e}=\frac{N_{e s p}}{S_{\text {dom }}} \int_{\Omega} N_{j}^{T} \cdot N_{i} \cdot \vec{e} d \Omega
$$

\section{Ensaio a vazio}

No EAV o transformador é alimentado com tensão $V_{\text {cir }}$ conhecida, impossibilitando a resolução individual da Equação (12), pois haveria duas incógnitas na equação do campo, $A$ e $I_{c i r}$. Para este caso, as equações do campo e do circuito elétrico, devem ser solucionadas em conjunto por meio do sistema apresentado na Equação (21) (ABBA E RACHEK, 2019).

$$
\left[\begin{array}{cc}
v[S] & -[D] \\
0 & {[R]}
\end{array}\right]\left\{\begin{array}{c}
A \\
I_{c i r}
\end{array}\right\}+\left[\begin{array}{cc}
{[T]} & 0 \\
{[E]} & 0
\end{array}\right] \frac{\partial}{\partial t}\left\{\begin{array}{c}
A \\
I_{c i r}
\end{array}\right\}-\left\{\begin{array}{c}
0 \\
V_{c i r}
\end{array}\right\}=\left\{\begin{array}{l}
0 \\
0
\end{array}\right\}
$$

A discretização no domínio do tempo é realizada conforme a Equação (22) (SALON, 1995):

$$
\beta \frac{\partial}{\partial t}\left\{\begin{array}{c}
A \\
I_{c i r}
\end{array}\right\}_{t}+(1-\beta) \frac{\partial}{\partial t}\left\{\begin{array}{c}
A \\
I_{c i r}
\end{array}\right\}_{t-\Delta t}=\frac{\left\{\begin{array}{c}
A \\
I_{c i r}
\end{array}\right\}_{t}-\left\{\begin{array}{c}
A \\
I_{c i r}
\end{array}\right\}_{t-\Delta t}}{\Delta t}
$$

onde $t$ é o instante de tempo, $\Delta t$ é o passo temporal e $\beta$ é o coeficiente que caracteriza o método de discretização empregado, para $\beta=1$ tem-se o Backward Difference (BDF). Dessa forma, de acordo com a Equação (22), para o método BDF, a Equação (21) torna-se:

$$
\left(\left[\begin{array}{cc}
v[S]+\frac{[T]}{\Delta t} & -[D] \\
\frac{[E]}{\Delta t} & {[R]}
\end{array}\right]\right)\left\{\begin{array}{c}
A \\
I_{c i r}
\end{array}\right\}_{t}-\frac{1}{\Delta t}\left[\begin{array}{cc}
{[T]} & 0 \\
{[E]} & 0
\end{array}\right]\left\{\begin{array}{c}
A \\
I_{c i r}
\end{array}\right\}_{t-\Delta t}-\left\{\begin{array}{c}
0 \\
V_{c i r}
\end{array}\right\}_{t}=\left\{\begin{array}{l}
0 \\
0
\end{array}\right\}
$$

Levando em consideração a relação não linear presente no núcleo do transformador, caracterizada pela curva BH da Figura 3, a solução da Equação (23) é realizada por meio do método de Newton-Raphson (N-R) (SALON, 1995), no qual um processo iterativo determina uma incógnita $x$ qualquer na iteração $\mathrm{k}+1$ a partir do seu valor na iteração $k$. O processo de iteração ocorre enquanto a variação $\Delta x=x^{k+1}-x^{k}$ for maior do que um erro $\varepsilon$. 
$\left(v\left(|\vec{B}|^{2}\right)[S]+\frac{\partial v}{\partial B^{2}}[P]+\frac{[T]}{\Delta t}\right)\{\Delta A\}_{t}^{k+1}=-\left(v\left(|\vec{B}|^{2}\right)[S]+\frac{[T]}{\Delta t}\right)\{A\}_{t}^{k}+\frac{[T]}{\Delta t}\{A\}_{t-\Delta t}+[D]\left\{I_{c i r}\right\}_{t}$

Após a resolução da Equação (27), a tensão aplicada a um enrolamento $V_{\text {cir }}$ pode ser obtida por meio da aplicação do método BDF na Equação (19), resultando em:

$$
\left\{V_{\text {cir }}\right\}_{t}=\frac{[E]}{\Delta t}\left(\{A\}_{t}-\{A\}_{t-\Delta t}\right)+[R]\left\{I_{\text {cir }}\right\}_{t}
$$

Os elementos da matriz $[R]$ devem considerar a resistência em série dos enrolamentos primário e secundário, indicados pelos sobrescritos $P$ e $S$, respectivamente, de forma que:

$$
R=\frac{N_{e s p}^{P} l^{P}}{\sigma_{e n r} S_{e s p}^{P}}+\left(\frac{N_{e s p}^{P}}{N_{e s p}^{S}}\right)^{2} \frac{N_{e s p}^{S} l^{S}}{\sigma_{e n r} S_{e s p}^{S}}
$$

Sendo generalizadas as equações (24) e (27) para uma malha completa (SADIKU, 2000).

\section{RESULTADOS}

Após a aplicação do MEF, extraem-se os resultados apresentados na Figura 4. Na Figura 4a está representada a tensão de curto-circuito no ECC, $V_{E C C}$, já na Figura $4 \mathrm{~b}$ observa-se a densidade de fluxo magnético no núcleo do transformador no EAV, $B_{E A V}$. As Figuras $4 \mathrm{c}$ e $4 \mathrm{~d}$ apresentam a corrente a vazio, $I_{E A V}$, em intervalos de tempo distintos. Enquanto na Figura 4c é possível observar um elevado valor de $I_{E A V}$ nos instantes iniciais, causado pela saturação do núcleo devido ao elevado valor de $B_{E A V}$, na Figura $4 \mathrm{~d}$ observa-se a estabilização de $I_{E A V}$ após um determinado período de tempo, seguindo o comportamento de $B_{E A V}$.

$\mathrm{Na}$ Tabela 1, os valores root mean square (RMS) de tensão $(V)$ e corrente $(I)$, potência ativa $(P)$ e os parâmetros do Quadro 1, extraídos da simulação por MEF são comparados aos dados medidos no transformador por meio do EAV e do ECC conforme Carvalho (2008). Os valores de tensão e corrente RMS e de $P$, na simulação da EAV, foram calculados com valores instantâneos obtidos entre 0,4 e $0,5 s$, onde é alcançado o regime permanente.

\section{CONSIDERAÇÕES FINAIS}

Os resultados da simulação do EAV, compilados na Tabela 1, apresentaram uma leve discrepância em relação aos valores medidos e tal ocorrido deve-se a não consideração acerca das perdas no ferro, fenômeno claramente conhecido. No EAV, a corrente $I_{E A V}$ obtida, representada na Figura $4 \mathrm{c}$, é a corrente de excitação $I_{\varphi}$ que percorre o núcleo do transformador, capturando a não linearidade característica do mesmo. A curva utilizada na simulação é representada na Figura 3, do material descrito como Silicon Steel M-6 Cross, sendo a que gerou resultados mais próximos aos medidos. Já os resultados da simulação do ECC apresentaram resultados próximos aos medidos, devendo-se ao fato de que no ECC o núcleo do transformador apresenta pouca influência sobre os parâmetros a serem calculados.

$\mathrm{Na}$ Figura $4 \mathrm{~b}$, o valor de $B_{E A V}$ nos instantes iniciais chega a 2,3 T, caracterizando a saturação do núcleo do transformador, resultando no alto valor inicial de $I_{E A V}$ representado na 
(C) COBENGE

"Os desafios para formar hoje o engenheiro do amanhã"
01 a 03 de dezembro Evento On-line

Figura 4c. Esse valor estabiliza-se em 1,5 T, e é refletido no comportamento da corrente a vazio, apresentada na Figura 4d.

A análise dos resultados obtidos por meio do MEF indica que o método permite determinar com moderada precisão os parâmetros do transformador no EAV e no ECC, assim como possibilita a medição da corrente de in-rush, Figura 4c, revelando-se um método útil para fins didáticos. $\mathrm{O}$ objetivo geral após a prática laboratorial seria alcançado, comparando as medições da simulação com as obtidas em laboratório.

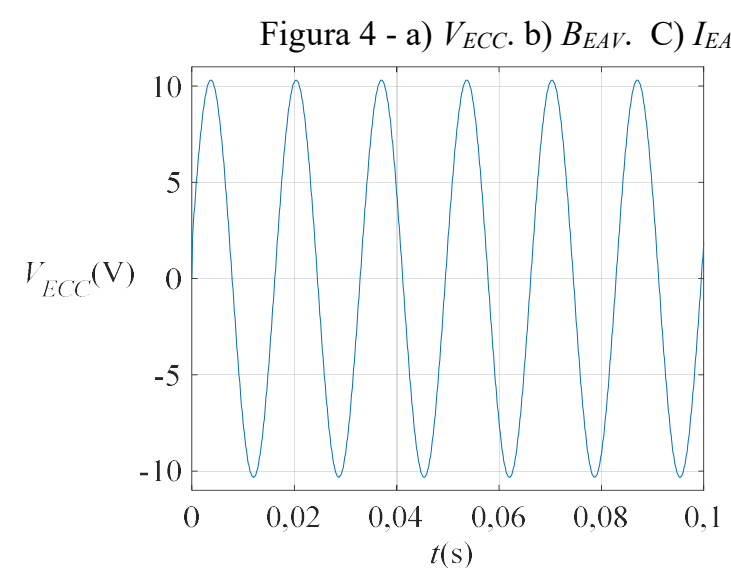

a)

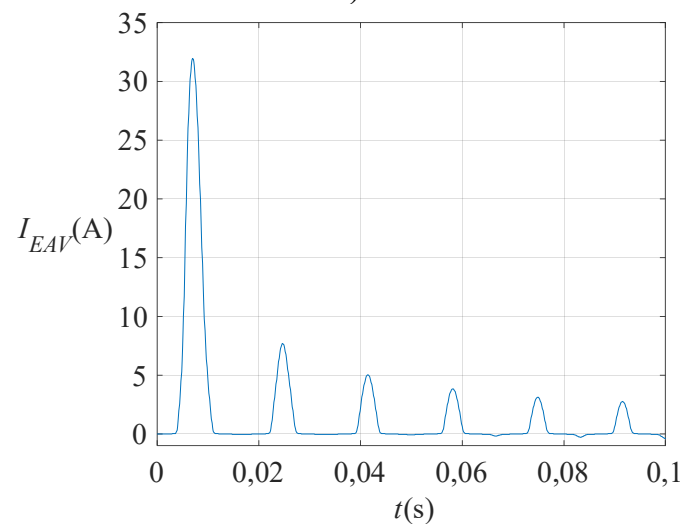

c)

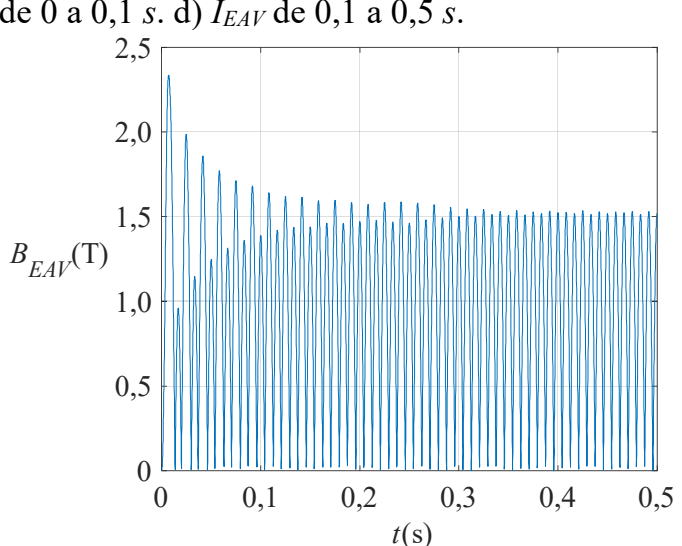

b)

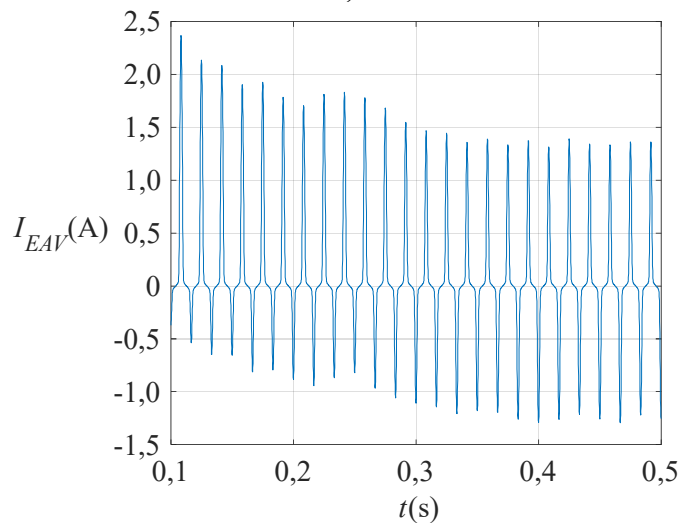

d)

Fonte: Autoria própria.

Tabela 1 - Resultados simulados e medidos dos parâmetros dos ensaios.

\begin{tabular}{cccccc}
\hline \multicolumn{3}{c}{ EAV } & \multicolumn{3}{c}{ ECC } \\
\hline Parâmetro & Simulação & Medição & Parâmetro & Simulação & Medição \\
\hline$V_{E A V}$ & $219,98 \mathrm{~V}$ & $219,8 \mathrm{~V}$ & $V_{E C C}$ & $7,29 \mathrm{~V}$ & $7,4 \mathrm{~V}$ \\
$I_{E A V}$ & $0,53 \mathrm{~A}$ & $0,62 \mathrm{~A}$ & $I_{E C C}$ & $0,72 \mathrm{~A}$ & $0,72 \mathrm{~A}$ \\
$P_{E A V}$ & $1,68 \mathrm{~W}$ & $5,1 \mathrm{~W}$ & $P_{E C C}$ & $5,16 \mathrm{~W}$ & $5,3 \mathrm{~W}$ \\
$R_{c}$ & $28804,29 \Omega$ & $9474,14 \Omega$ & $R_{e q}$ & $9,95 \Omega$ & $10,22 \Omega$ \\
$X_{m}$ & $415,1 \Omega$ & $354,74 \Omega$ & $X_{e q}$ & $1,85 \Omega$ & $1,01 \Omega$ \\
\hline \multicolumn{5}{c}{ Fonte: Autoria Própria. }
\end{tabular}

\section{Agradecimentos}

Ao professor Antônio Sérgio Bezerra Sombra, professor visitante na UFERSA, por disponibilizar instalações e recursos computacionais do LOCEM/UFC para a realização deste trabalho. 


\section{REFERÊNCIAS}

ABBA, Faiza; RACHEK, M'hemed. Time-Stepping FEM-Based Multi-Level Coupling of Magnetic Field-Electric Circuit and Mechanical Structural Deformation Models Dedicated to the Analysis of Electromagnetic Actuators. Disponível em: https://www.mdpi.com/2076-0825/8/1/22/pdf-vor. Acesso em: 05 jul. 2020.

BASTOS, João Pedro A.; SADOWSKI, Nelson. Electromagnetic Modeling by Finite Element Methods. New York: CRC Press. 2003.

BASTOS, João Pedro A.; SADOWSKI, Nelson. Magnetic Materials and 3D Finite Element Modeling. New York: CRC Press. 2013.

BÍRÓ, Oszkár; PREIS, Kurt; PAUL, Christian. The use of a reduced vector potential $\mathrm{A}_{r}$ formulation for the calculation of iron induced field errors. ROXIE: routine for the optimization of magnet $X$-sections, inverse field calculation and coil end design, $p$. 31-46, 1999.

CARVAlHO, Geraldo. Máquinas Elétricas Teoria e Ensaios. São Paulo: Editora Érica. 2008.

ELMÔR FILHO, Gabriel et al. Um Nova Sala de Aula é Possível: Aprendizagem Ativa na Educação em Engenharia. Rio de Janeiro: LTC. 2019.

RENYUAN, Tang; SHENGHUI, Wang; YAN, Li; XIULIAN, Wang; XIANG, Cui. Transient simulation of power transformers using 3D finite element model coupled to electric circuit equations. IEEE Transactions on Magnetics, vol. 36, no. 4, p. 1417-1420, 2000.

SADIKU, Matthew N.O. Numerical Techniques in Electromagnetics. New York: CRC Press. 2000.

SALON; S. J. Finite Element Analysis of Electrical Machines. Netherlands: Editora Springer. 1995.

\section{SIMULATIONS BY FINITE ELEMENTS APPLIED TO TESTS OF TRANSFORMER IN OUTSIDE CLASS OF LABORATORY PRACTICE}

Abstract: In this article the application of the finite element method for the calculation of parameters of a transformer is presented, which it was previously analyzed by means of experimental procedures, also called tests. In the presented method, the connection with electrical circuits, the time domain simulations, as well as material characteristics of the transformer core are taken into account in the finite element modeling. Such simulations shown good precision and the tests provide data and the comparison between simulation results and experimental ones can be performed, showing the inverted classroom background and the motivation for predicting the experimental results.

Keywords: Inverted classroom, no-load test, short-circuit test, 3D simulation. 